\title{
Práticas educativas realizadas pelo enfermeiro à pessoa com estomia
}

\author{
Sara Rodrigues Rosado, M.Sc.*, Walter Batista Cicarini, M.Sc.**, Cibelle Barcelos Filipini, M.Sc.***, \\ Rogério Silva Lima, M.Sc. ${ }^{* * * *}$, Eliza Maria Rezende Dázio, D.Sc.******
}

\begin{abstract}
Enfermeira, Professora da Universidade Federal de São João Del-Rei, **Bioquímico, Doutorando em Análises Clínicas na Escola de Farmácia da Universidade Federal de Minas Gerais (UFMG), ***Enfermeira, Enfermeira do Centro Especializado em Reabilitação de Alfenas/MG, ****Enfermeiro, Professor do Curso de Medicina da Universidade Federal de Alfenas (UNIFAL$M G),{ }^{* * * *}$ Enfermeira, Professora do Curso de Graduação e Pós-Graduação em Enfermagem da UNIFAL-MG
\end{abstract}

\section{Resumo}

Objetivo: Analisar as produçōes científicas nacionais e internacionais sobre as práticas educativas em saúde desenvolvidas pelo enfermeiro às pessoas com estomia. Método: Revisão integrativa com vista a responder a questão: Quais são as práticas educativas em saúde realizadas pelo enfermeiro às pessoas com estomia? Foi realizada busca de artigos publicados de 2003 a 2013, utilizando o instrumento de Chaves e as bases de dados Lilacs, Web of Science, Medline, Ibecs, Pubmed, BDENF e bibliotecas virtuais Scielo. Resultados: Cinco artigos selecionados direcionam as açóes de educaçáo em saúde pautadas no diálogo e utilização de materiais educativos (programa de multimídia e cartilha) que auxiliaram no processo de reabilitação. Conclusão: As açóes educativas dos enfermeiros devem ser desenvolvidas desde o período pré-operatório e estender-se ao domicílio, sendo imprescindível a elaboraçáo de um plano de educaçáo individualizado embasado nas práticas e saberes da pessoa com estomia respeitando sua realidade social e cultural.

Palavras-chave: educação em saúde, Enfermagem, estomia.

\section{Abstract}

\section{Educational health practices developed by nurses for people with ostomy}

Objective: To analyze national and international scientific publications on educational health practices developed by nurses for people with ostomy. Method: This is an integrative literature review that aimed to answer the question: Which are the health education actions performed by nurses for people with ostomy? Studies published from 2003 to 2013 were searched using a Chaves instrument and Lilacs, Web of Science, Medline, Ibecs, Pubmed, BDENF and Scielo data bases. Results: We identified five studies which focused the actions to promote health education based on dialogue and use of educational materials (multimedia program and booklet) and to help in the rehabilitation process. Conclusion: The educational activities of nurses should be developed during the pre-operative period and extended at home. It is imperative to create an individualized education plan based on practices and knowledge of people with ostomy respecting their socio-cultural reality.

Key-words: health education, Nursing, ostomy. 


\section{Resumen}

\section{Prácticas educativas desarrolladas por el enfermero a persona con estoma}

Objetivo: Analizar las publicaciones científicas nacionales e internacionales sobre las prácticas educativas en salud desarrolladas por enfermeros para personas con estoma. Método: Se trata de revisión integradora de la literatura que tuvo como objetivo responder a la siguiente pregunta: ¿Cuáles son las prácticas educativas en salud realizadas por los enfermeros para las personas con estoma? Para la búsqueda de estudios publicados desde 2003 hasta 2013 se utilizó un instrumento de Chaves y las bases de datos Lilas, Web of Science, Medline, Ibecs, Pubmed, BDENF y Scielo. Resultados: Cinco estudios seleccionados tienen como objetivo acciones de educación en salud basados en el diálogo y el uso de materiales educativos (programas multimedia y folleto) que colaboraron en el proceso de rehabilitación. Conclusión: Las actividades educativas de los enfermeros deberían desarrollarse a partir del período pre-operatorio y extenderse al domicilio. Es imprescindible la elaboración de un plan de educación individualizado basado en las prácticas y conocimientos de personas con estoma respetando su realidad social y cultural.

Palabras-clave: educación en salud, Enfermería, estomía.

\section{Introdução}

A educação em saúde deve ser vislumbrada como uma forma de estimular a população a analisar de forma crítica sua realidade, determinar suas próprias metas de saúde, compreender sobre o processo de saúde-doença e, também, elaborar estratégias de intervenção e modificar situaçóes vigentes [1]. Assim, o diálogo, a troca de informaçóes e de conhecimentos são fatores importantes no intuito de esclarecer as principais dúvidas das pessoas com estomia e fomentar sua participação no processo educativo [1].

O cuidar em enfermagem deve ultrapassar o olhar reducionista de assistir ao doente e direcionar o seu foco de atençáo à saúde por meio da compreensão dos sujeitos em uma perspectiva holística. Neste sentido, a promoçáo da saúde e a educaçáo em saúde encontram-se entrelaçados, com vistas a promover e melhorar a qualidade de vida de toda a populaçáo e náo apenas das pessoas sob o risco de adoecer $[1,2]$.

Para o enfermeiro, a educação em saúde constitui-se como um instrumento fundamental para uma assistência de qualidade, pois o enfermeiro é também educador ao realizar suas orientaçóes, seja para o paciente ou para a família [3]. No âmbito da educação em saúde, a enfermagem tem como objetivo propiciar aos usuários dos serviços um maior aprendizado sobre o autocuidado, além de formar multiplicadores de conhecimento [3]. Educar as pessoas, sobretudo aquelas que possuem uma estomia - abertura cirúrgica, definitiva ou provisória de um órgão, formando uma boca que fica em contato com o meio externo para alimentação, eliminaçóes de secreções como fezes e/ou urina [4] —, não deve ser concebido como uma forma de depositar conhecimentos e valores, mas sim como ato cognoscente, centrado na problematização do cotidiano, na experiência individual, familiar e de grupos sociais, e no respeito às diferenças culturas [5].

O processo de educar as pessoas com estomia tem como propósito contribuir para seu autocuidado e sua autoformação, ensinando-as a assumir sua nova condição e ajudando-as a viver de forma independente. A promoçáo de saúde tem como um dos campos de ação o estímulo ao desenvolvimento de atitudes e habilidades pessoais positivas à saúde, para isso, é indispensável coletar informaçóes sobre a educaçáo para a saúde, no lar, na escola, no trabalho e nos demais espaços coletivos [6].

$\mathrm{O}$ processo ensino-aprendizagem da pessoa com estomia deve ser iniciado na fase pré-operatória, nesse momento a pessoa e sua família precisam ser esclarecidas sobre a cirurgia que resultará na alteração da imagem corporal [7]. Em relação à alta hospitalar, esta fase exige da família disponibilidade; recursos financeiros para o tratamento; conhecimento dos serviços públicos para atendimento das possíveis complicaçóes, entre outros fatores [8]. Por isso, a transição para o domicílio deve ser planejada por uma equipe multidisciplinar e a participação dos familiares, a fim de garantir o prosseguimento do cuidado com qualidade.

Entretanto, a aprendizagem após a alta e as açôes educativas devem ter continuidade no domi- 
cílio por meio de grupos de apoio cujo objetivo seja a reabilitação e a reinserção social, de forma que o paciente e sua família encontrem maneiras de viver com qualidade [3].

Durante nossa prática profissional, percebemos que as pessoas com estomia recebiam a alta hospitalar sem uma assistência adequada sobre sua nova condição. Quando algo era realizado, restringia-se a um treinamento incipiente sobre as técnicas de limpeza e troca de bolsa coletora, sem se atentar para as reais necessidades individuais. Neste contexto e diante da escassez de estudos sobre esta temática, principalmente relacionada à educação em saúde oferecida a estes clientes, decidimos entáo realizar esta revisáo integrativa com objetivo de analisar as produçóes científicas nacionais e internacionais sobre as práticas educativas em saúde desenvolvidas pelo enfermeiro às pessoas com estomia.

\section{Material e métodos}

Trata-se de um estudo bibliográfico e documental efetuado por meio da revisão integrativa da literatura. Esse método de pesquisa permite a análise de estudos relevantes que dáo suporte para a tomada de decisão e a melhoria da prática clínica. Tem como finalidade reunir e sintetizar resultados de pesquisas sobre um determinado tema ou questão, de maneira sistemática e ordenada, contribuindo para o aprofundamento de conhecimento do tema investigado [9].

A pesquisa pautou-se pela seguinte questão norteadora: quais são as práticas educativas em saúde realizadas pelo enfermeiro às pessoas com estomia?

Foram exploradas bases de dados eletrônicas da BVS: Literatura Latino-Americana e do Caribe através de Informaçóes em Ciências da Saúde (Lilacs), Medline, BDENF, Central - Registro de ensaios clínicos controlados, IBECS, Web of Science e a biblioteca virtual Scientific, Electronic Library Online (Scielo). Para empreender a busca foram utilizados os Descritores em Ciências da Saúde (DeCS) associados em português "Educação em saúde" and "Enfermagem" and "Estomia",

Adotou-se como critério de inclusão os artigos em Português, Espanhol e Inglês, disponíveis na íntegra, produzidos no período de 2003 a setembro 2013. Foram excluídas teses, dissertações, livros, relatórios de conferências, resumos de congresso e artigos que não respondessem a questão norteadora.
A busca resultou em 60 publicaçóes indexadas, entretanto, apenas 15 artigos atendiam aos critérios de inclusão. Desse total, foram excluídas 7 publicaçôes duplicadas. Os oito (8) trabalhos restantes foram submetidos à leitura do título e do resumo. Após leitura cuidadosa destes, observou-se que três (03) trabalhos não respondiam à questão norteadora. Portanto, a amostra constituiu-se de cinco (5) publicaçóes que foram analisadas detalhadamente.

Para a análise dos artigos selecionados utilizou-se um instrumento adaptado de Chaves [10] que favoreceu a identificação e organização dos seguintes elementos dos estudos: identificação do artigo, ano, autor, país de publicação, periódico de publicação, país de estudo, objetivo do estudo, resultados e conclusóes do estudo.

Em seguida, os estudos foram classificados quanto ao nível de evidência, baseando-se na classificação de Melnyk e Fineout-Overholt [11].

A análise dos dados foi realizada em duas etapas. A primeira etapa se constitui na síntese dos dados, identificação e caracterização da amostra. A segunda etapa foi composta pela análise do conteúdo dos artigos [12].

\section{Resultados}

Os cinco artigos resultantes do processo de revisão foram publicados no período de 2010 a 2012, em periódicos nacionais e internacionais, na língua inglesa e portuguesa, no Brasil e Taiwan, sendo que todos foram publicados em revistas específicas de Enfermagem (Tabela I).

Quanto à abordagem metodológica escolhida e método de investigação, quatro estudos foram qualitativos, três destes se destacam por utilizar como referencial teórico-metodológico a Pedagogia de Paulo Freire; um trabalho foi com abordagem quantitativa e estudo controlado randomizado duplo-cego. Apenas um estudo atendeu ao nível de evidência II e os demais foram V (Tabela II).

Os artigos abordam em sua temática a educação em saúde dos enfermeiros para as pessoas com estoma, por meio do diálogo de um grupo e de recursos audiovisuais como programa de multimídia e cartilha sobre os cuidados com estomia (Tabela III).

\section{Discussão}

No que se refere às práticas educativas em saúde realizadas pelo enfermeiro às pessoas com 
Tabela I - Distribuição dos artigos por país de estudo, periódico, ano e língua de publicação. Alfenas/MG, 2013.

\begin{tabular}{llllcl}
\hline ID & \multicolumn{1}{c}{ Autores } & \multicolumn{1}{c}{ Periódico de publicação } & País do estudo & $\begin{array}{c}\text { Ano de } \\
\text { publicação }\end{array}$ & Idioma \\
\hline A1 & Martins PAF, Alvim NAT [13] & Revista Brasileira de Enfermagem & Brasil & 2010 & Português \\
A2 & Barros EJL et al. [14] & Revista Gaúcha Enfermagem & Brasil & 2012 & Português \\
A3 & Cunha RR et al. [15] & Acta Paulista Enfermagem & Brasil & 2012 & Português \\
A4 & Martins PAF, Alvim NAT [16] & Texto Contexto Enfermagem & Brasil & 2012 & Português \\
A5 & Lo SF et al. [17] & Journal of Advanced Nursing & Taiwan & 2010 & Inglês \\
\hline
\end{tabular}

Tabela II - Distribuição dos artigos conforme abordagem metodológica. Alfenas/MG, 2013.

\begin{tabular}{clc}
\hline ID & \multicolumn{1}{c}{ Abordagem metodológica } & $\begin{array}{c}\text { Nível de } \\
\text { evidência }\end{array}$ \\
\hline A1 & $\begin{array}{l}\text { Abordagem metodológica qualitativa, método de investigação Pesquisa Convergente-Assistencial } \\
\text { e a base teórica a pedagogia freiriana aplicada à enfermagem. }\end{array}$ & $\mathrm{V}$ \\
A2 & $\begin{array}{l}\text { Abordagem metodológica qualitativa. } \\
\text { A3 }\end{array}$ & $\begin{array}{l}\text { Abordagem metodológica qualitativa em saúde articulada com itinerário de pesquisa freireana. } \\
\text { Abordagem metodológica qualitativa e método de investigação pesquisa convergente-assisten- }\end{array}$ \\
A4 & $\begin{array}{l}\text { cial e a base teórica a pedagogia freiriana. } \\
\text { Abordagem metodológica quantitativa e método de investigação estudo controlado randomiza- } \\
\text { do duplo-cego. }\end{array}$ & $\mathrm{V}$ \\
$\mathrm{A} 5$ & $\mathrm{I}$ \\
\hline
\end{tabular}

Tabela III - Síntese dos estudos selecionados nas bases de dados. Alfenas/MG, 2013.

\begin{tabular}{|c|c|c|}
\hline ID & Principais resultados sobre as práticas educativas & Conclusão \\
\hline A1 & $\begin{array}{l}\text { Revelaram-se alguns saberes e práticas na prática dia- } \\
\text { lógica entre enfermeira-usuário. Nesta troca envolvente, } \\
\text { emergiram práticas e saberes acerca da troca da bolsa } \\
\text { coletora (drenável e não-drenável); manutenção diária } \\
\text { da bolsa coletora drenável; irrigação; uso de adjuvan- } \\
\text { tes; complicações tardias (dermatite e prolapso); vida } \\
\text { social, familiar e laborativa; religiosidade; sexualidade; } \\
\text { exercícios e desportos; lazer; vestuário e dieta. }\end{array}$ & $\begin{array}{l}\text { É importante dialogar com a pessoa com estomia } \\
\text { sobre seus saberes e práticas de modo a criar possibi- } \\
\text { lidades de integração destes com aqueles desenvolvi- } \\
\text { dos no contexto ambulatorial. Para tanto, sua parti- } \\
\text { cipação é imprescindível, pois favorece a crítica e a } \\
\text { reflexão e, por conseguinte, a tomada de consciência } \\
\text { sobre os caminhos que podem favorecer ou prejudicar } \\
\text { a sua saúde e bem-estar. }\end{array}$ \\
\hline A2 & $\begin{array}{l}\text { A cartilha educativa foi elaborada com auxílio de idosos } \\
\text { com estomia e composta por categorias de forma a } \\
\text { facilitar a compreensão: direitos dos estomizados; } \\
\text { conceitos e tipos de estomas; cuidados com a estomia } \\
\text { e importância da família e do grupo de apoio para o } \\
\text { cuidado. }\end{array}$ & $\begin{array}{l}\text { A cartilha educativa apresenta-se como um produto } \\
\text { gerontotecnológico útil para o cuidado ao idoso esto- } \\
\text { mizado à luz da complexidade. É um instrumento que } \\
\text { articula o saber técnico às questões humanas, com a } \\
\text { finalidade de humanizar o cuidado pelo enfermeiro. }\end{array}$ \\
\hline A3 & $\begin{array}{l}\text { A assistência de enfermagem à pessoa estomizada } \\
\text { não é proporcionada de maneira dialógica e tem a } \\
\text { ausência de eficácia nas orientações sobre o pré e pós- } \\
\text {-cirúrgicos o que ocasiona dificuldades de adaptação } \\
\text { dessa nova realidade e, assim, prolongando o período } \\
\text { de reabilitação. } \\
\text { A oportunidade de serem ouvidos e poderem juntos } \\
\text { sugerir ações de transformação da realidade vivenciada } \\
\text { favoreceu aos participantes desvelarem coletivamente a } \\
\text { proposta de implantação de um Programa de Educação } \\
\text { Permanente na atenção à Pessoa Estomizada nos hos- } \\
\text { pitais públicos e privados, para profissionais de saúde } \\
\text { com participação do estomizado. }\end{array}$ & $\begin{array}{l}\text { O diálogo revelou que essas pessoas vivenciam } \\
\text { diferentes períodos de adaptação até a reabilitação; } \\
\text { que expressam suas necessidades e demandas refe- } \\
\text { rentes ao tratamento cirúrgico. Assim, a participação } \\
\text { no processo decisório foi relevante não apenas aos } \\
\text { participantes do grupo, pois, à medida que exercia o } \\
\text { diálogo, era possível sentir-se parte do grupo e corres- } \\
\text { ponsáveis. }\end{array}$ \\
\hline
\end{tabular}




\begin{tabular}{|c|c|c|}
\hline $\mathrm{A} 4$ & $\begin{array}{l}\text { A partir das vivências e experiências de clientes sobre a } \\
\text { manutenção da estomia que se revelaram no encontro } \\
\text { com a enfermeira construiu-se um plano de cuidados } \\
\text { individual. Emergiram práticas e saberes acerca da tro- } \\
\text { ca da bolsa coletora (drenável e fechada); manutenção } \\
\text { diária da bolsa coletora drenável (uma abertura e duas } \\
\text { aberturas); manutenção da bolsa coletora fechada; } \\
\text { manutenção diária da bolsa coletora drenável para } \\
\text { urostomia; autoirrigação; uso de adjuvantes (barreira } \\
\text { protetora de pele em pasta e pó, e lubrificante deso- } \\
\text { dorante); complicações tardias (dermatite, prolapso } \\
\text { e hérnia); uso de acessórios (cinta e adesivo fixador); } \\
\text { banho de sol (falta de privacidade para o banho de } \\
\text { sol e falta de tempo para o banho de sol); proteção do } \\
\text { equipamento durante o banho habitual; vida social, } \\
\text { familiar e laborativa; religiosidade e espiritualidade; } \\
\text { sexualidade; exercícios e desportos; lazer; direitos e } \\
\text { deveres; vestuário e dieta. } \\
\text { A educação em saúde revelou-se como uma chave para } \\
\text { o sucesso na convivência do cliente com o estoma. Esse } \\
\text { cliente, consciente dos seus limites e possibilidades para } \\
\text { o cuidado de manutenção da sua estomia de elimina- } \\
\text { ção, torna-se um aliado na relação de cuidado. }\end{array}$ & $\begin{array}{l}\text { A pedagogia freireana revelou-se como um instrumen- } \\
\text { to facilitador na aprendizagem de clientes estomizados } \\
\text { no âmbito de uma proposta educativa do cuidado de } \\
\text { enfermagem no contexto ambulatorial. Problematizan- } \\
\text { do as diferentes situações que se lhes apresentam na } \\
\text { convivência com a estomia de eliminação, os clientes } \\
\text { foram levados a refletir sobre sua realidade através } \\
\text { da prática dialógica implementada na convergência } \\
\text { pesquisa-cuidado. } \\
\text { Com o retorno dos clientes no ambulatório, após a } \\
\text { implementação do cuidado compartilhado, foi possível } \\
\text { denotar os sucessos derivados da pedagogia utilizada } \\
\text { no processo educativo. Sua condição de crítica e de } \\
\text { reflexão está aguçada; exercendo com maior seguran- } \\
\text { ça e autonomia os cuidados relacionados à manuten- } \\
\text { ção de sua estomia, avaliando, modificando hábitos, } \\
\text { transformando a realidade. }\end{array}$ \\
\hline A5 & $\begin{array}{l}\text { A intervenção programa de educação multimídia (MEP) } \\
\text { melhorou significativamente os níveis de conhecimento } \\
\text { dos pacientes com estomia, e esse conhecimento está } \\
\text { ligado a melhores atitudes de autocuidado. } \\
\text { Ele também demonstra que os pacientes são capazes } \\
\text { de absorver informação e formar crenças de autoaten- } \\
\text { dimento no período imediatamente após a cirurgia, que } \\
\text { muitas vezes torna-se confuso e complexo para esta } \\
\text { pessoa. }\end{array}$ & $\begin{array}{l}\text { Empregando uma intervenção educacional através } \\
\text { de uma abordagem multimídia, no início do período } \\
\text { pós-operatório pode ter um efeito positivo sobre os } \\
\text { níveis de conhecimento e também promover atitudes } \\
\text { de autocuidado e comportamento. } \\
\text { Tendo em conta estes resultados, os formuladores de } \\
\text { políticas devem considerar substituição de informa- } \\
\text { ções por escrito por programas de multimídia como } \\
\text { parte da educação pós-operatório dos pacientes com } \\
\text { estoma. }\end{array}$ \\
\hline
\end{tabular}

estomia, os cinco estudos apontam como instrumentos o diálogo entre profissional-paciente e a utilização de materiais educativos no processo de reabilitação.

Para o sucesso destas ações em saúde, utilizaram uma cartilha elaborada pelos próprios idosos com estomia cadastrados no serviço [14]. Essa cartilha foi estruturada pelos temas: direitos dos estomizados; conceitos e tipos de estomas; cuidados com a estomia e importância da família e do grupo de apoio para o cuidado.

A cartilha educativa apresentou-se como um produto gerontotecnológico útil para o cuidado destes idosos e é um instrumento que articula recursivamente as questôes técnicas com as humanas, com a finalidade de humanizar o cuidado do enfermeiro ao idoso estomizado [12].
Outros pesquisadores utilizaram para a educação em saúde, recursos audiovisuais como o Programa de Educação Multimídia (MEP) [17]. Este programa ampliou de forma significativa o conhecimento dos pacientes com estomia. Torna-se relevante destacar que a ampliaçáo de conhecimento está ligada a atitudes de autoconfiança e autocuidado que favorecem mudanças de comportamento e manutenção em saúde [17].

Em relação ao referencial teórico, observou-se que três artigos utilizaram a pedagogia de Paulo Freire como forma de abordar as pessoas com estoma. Este tipo de método pedagógico valoriza uma abordagem crítico-reflexiva por meio do diálogo entre profissionais e pacientes sempre respeitando seus saberes, práticas e cultura, bem como a troca de experiência $[13,15,16]$. 
Percebeu-se que o eixo condutor do diálogo pautou-se na necessidade de melhor orientação antes e após a cirurgia geradora de estomia. A codificação/descodificação evidenciou que à pessoa com estomia náo é proporcionada, de maneira dialógica, a orientação necessária antes e após a cirurgia, ocasionando dificuldades de adaptação dessa nova realidade e, assim, prolongando o período de reabilitação [15].

Quanto ao conteúdo abordado nos encontros, destacaram-se alguns saberes e práticas acerca do manejo do equipamento coletor (drenável e não-drenável) como sua troca a cada três dias, sua retirada de cima para baixo sob o chuveiro e o modo de recortar e inserir o dispositivo; alguns aspectos relacionados aos cuidados com o estoma, como a higienização com sabão neutro e água, secagem com toalha de maneira suave sobre a pele periestomal [13]. A dermatite foi uma complicação mencionada em maior escala cujas causas foram diversas: troca da bolsa coletora constantemente, fricçáo exagerada com gaze para retirada de cola sobre a pele, má adaptação aos equipamentos coletores, recorte da base adesiva de forma inapropriada e tratamentos com os adjuvantes (pó e pasta) que utilizados indevidamente [16]. A manutenção diária do equipamento coletor drenável foi também apontada como tema pelas pessoas com estomia, além de irrigação da colostomia; vida social, familiar e laborativa; religiosidade; sexualidade; exercícios e desportos; lazer; vestuário e dieta [13].

Destaca-se como frutos da educação em saúde a proposta dos autores de implementar um Programa de Educação na atenção à Pessoa Estomizada nos hospitais públicos e privados para profissionais de saúde que prestem assistência às pessoas com estomias [15].

\section{Conclusão}

Este estudo possibilitou a análise das produçóes científicas nacionais e internacionais sobre as práticas educativas em saúde desenvolvidas pelo enfermeiro às pessoas com estomia. Observou-se a escassez de estudos no período estudado. Os artigos em sua maioria caracterizaram-se por baixo nível de evidência.

Em relação às práticas educativas em saúde realizadas pelo enfermeiro às pessoas com estomia, os estudos se referem ao diálogo entre profissional-paciente e à utilização de materiais educativos no processo de reabilitação, entre eles programa de multimídia e cartilha. Valorizam ainda a troca de experiência entre os pacientes. Quanto ao conteúdo abordado nos encontros destacam-se: troca e manutenção do equipamento coletor; complicaçóes tardias e tratamento com adjuvantes; irrigação; uso de acessórios; vida social, familiar e laborativa; religiosidade e espiritualidade; sexualidade; atividade física; lazer; direitos e deveres; vestuário e dieta.

As açóes educativas dos enfermeiros devem ser desenvolvidas desde o período pré-operatório e estender-se após a alta hospitalar, de forma contínua no domicílio, sendo imprescindível a elaboração de um plano de educação individualizado embasado nas práticas e saberes da pessoa com estomia respeitando sua realidade social e cultural.

O processo educativo, quando embasado na abordagem crítico-reflexiva, envolve o paciente na construção de seu conhecimento, de forma a valorizar sua subjetividade, identificar possíveis barreiras e anseios, e elaborar recursos para enfrentá-los a fim de tornar as açôes de educação em saúde mais eficazes.

Nesse sentido, considera-se a necessidades de pesquisas pautadas em abordagens metodológicas que possibilitem a verificação da efetividade das açóes educativas do enfermeiro na melhoria da qualidade de vida das pessoas estomizadas.

\section{Referências}

1. Ceccon RF, Oliveira KM, Rossetto MS, Germani ARM. Educação em saúde: percepção de profissionais atuantes em uma coordenadoria regional de saúde. Rev Gaúch Enferm 2011;32:56-62.

2. Souza MTM, Silva MD, Carvalho M. Revisão integrativa: o que é e como fazer. Einstein (São Paulo) 2010;8:102-6.

3. Reveles AG, Takahashi RT. Educação em saúde ao ostomizado: um estudo bibliométrico. Rev Esc Enferm USP 2007;41:245-50.

4. Delavechia RP. A percepção de si como ser-estomizado: um estudo fenomenológico. Rev Enferm UERJ 2010;18:223-8.

5. Alves GG, Aerts D. As práticas educativas em saúde e a Estratégia Saúde da Família. Ciênc Saúde Coletiva 2011;16:319-25.

6. Casarin MR, Piccoli JC. E. Educação em saúde para prevenção do câncer de colo do útero em mulheres do município de Santo Ângelo/RS. Ciênc Saúde Coletiva $2011 ; 16: 3925-32$.

7. Melaragno ALP. Estomas em pediatria - peculiaridades do cuidado. In: Matsubara MGS. Feridas e estomas em oncologia: uma abordagem interdisciplinar. São Paulo: Lemar; 2012. p. 195-199. 
8. Barreto LCL, Cardoso MHCA, Villar MAM, Gilbert ACB. Percepçóes dos profissionais de uma unidade de internação pediátrica sobre a alta de crianças ostomizadas. Rev Gaúch Enferm 2008;29:438-45.

9. Mendes KDS, Silveira RCP, Galvão CM. Revisão integrativa: método de pesquisa para a incorporaçấo de evidências na saúde e na enfermagem. Texto Contexto-Enferm 2008; 17:758-64.

10. Chaves ECL. Revisão do diagnóstico de enfermagem angústia espiritual [Tese]. Ribeirão Preto: Escola de Enfermagem de Ribeirão Preto, Universidade de São Paulo; 2008.

11. Melnyk BM, Fineout-Overholt E. Evidence based practice in nursing $\&$ healthcare: a guide to best practice. 2 nd ed. Philadelphia: Wolters Kluwer Health/Lippincot Williams \& Wilkins; 2011.

12. Campos RG. Bournout: uma revisão integrativa na enfermagem oncológica [Dissertação]. Ribeirão Preto:
Escola de Enfermagem de Ribeirão Preto, Universidade de São Paulo; 2005.

13. Martins PAF, Alvim NAT. Perspectiva educativa do cuidado de enfermagem sobre a manutenção da estomia de eliminação. Rev Bras Enferm 2010;64:322-7.

14. Barros EJL, Santos SSC, Gomes GC, Erdmann AL. Gerontotecnologia educativa voltada ao idoso estomizado à luz da complexidade. Rev Gaúch Enferm 2012;33:95-101.

15. Cunha RR, Backes VMS, Heidemann ITSB. Desvelamento crítico da pessoa estomizada: em ação o programa de educaçáo permanente em saúde. Acta Paul Enferm 2012;25:296-301.

16. Martins PAF, Alvim NAT. Plano de cuidados compartilhado junto a clientes estomizados: a pedagogia freireana e suas contribuiçóes à prática educativa da enfermagem. Texto Contexto Enferm 2012;21:286-94.

17. Lo SF, Wang YT, Wu LY, Hsu MY, Chang SC, Hayter M. Multimedia education programme for patients with a stoma: effectiveness evaluation. J Adv Nurs 2010;67:68-76. 